\title{
DC COLLECTION NETWORKS FOR OFFSHORE GENERATION
}

\author{
Yiqing Lian*, Stephen J. Finney ${ }^{\dagger}$ \\ ${ }^{\dagger}{ }^{\dagger}$ Wind Energy CDT, University of Strathclyde, Glasgow, United Kingdom \\ yiqing.lian@strath.ac.uk, stephen.finney@strath.ac.uk
}

Keywords: offshore wind energy, DC grid, DC-DC converter

\begin{abstract}
Onshore wind farms can now be regarded as a mature technology, capable of providing increasing levels of clean energy. The development of offshore wind technology will provide the ability to harness much larger wind energy resource. Offshore wind arrays present many new challenges including the electrical power system which provides the internal collection system and the connection to the on-shore power network. For remote offshore wind farms, high voltage direct current (HVDC) transmission will be required to transmit power from the wind farm to the shore. The use of HVDC has the effect of decoupling the wind farms internal collection network from the rest of the power grid, thereby removing the requirement for a conventional alternating current (AC) network. This paper discusses the use of a direct current (DC) collection system for offshore wind farms, with particulars emphasis of DC-DC converter requirements. The proposed converter is validated by the simulation model and the performances e.g. switching losses, conduction losses are investigated.
\end{abstract}

\section{Introduction}

Compared to the conventional fossil fuel sources, major advantages with the use of renewable energy are to reduce the impact of climate-change with their environmentally friendly nature, offer sustainable sources and bring economic benefits. Among various renewable technologies, the environmental benefits and cost-competitiveness have driven the rapid expansion of wind power as a significant green source in recent decades.

In March 2007, the EU endorsed a strategic energy technology plan which sets a target of $20 \%$ of the energy needs to come from the renewable sources by 2020 to increase the share of the renewable energy sectors [1]. Included in the plan, UK has embarked on a strategy aimed at providing $15 \%$ of energy consumed via renewable sources and $20 \%$ of the electricity via renewables by 2020 [2]. And wind energy is regarded as a significant contributor to meet the target. In china, starting from nearly zero share in 2004 , China has made an aggressive plan of wind power development and by far China has led the world, installing $13 \mathrm{GW}$ in 2012 to bring the total so far to $77 \mathrm{GW}$ installation and intended to have $230 \mathrm{GW}$ wind capacity by 2020 [3]. As reported by Global Wind Energy Council (GWEC), wind power was expanded by almost $20 \%$ in 2012 across the world to reach a new peak of $282 \mathrm{GW}$ of total installed capacity [5]. And there is an expected substantial growth in wind energy capacity around the world in the coming years.

Global Cumulative Installed Wind Capacity 1998-2012

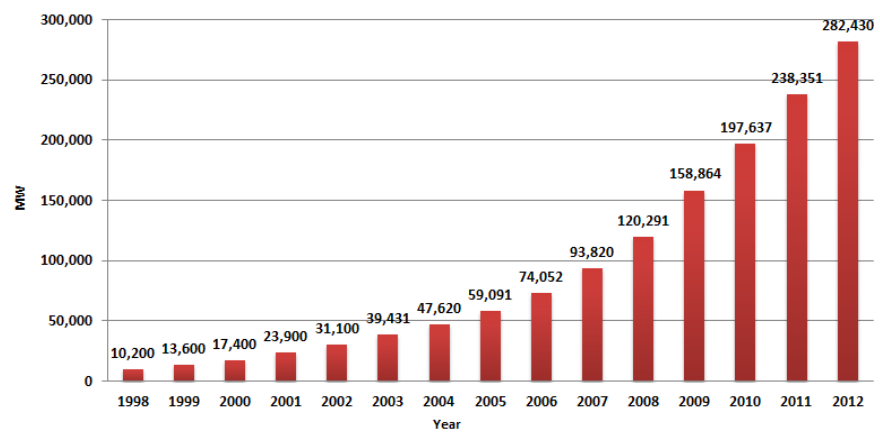

Figure 1: Global cumulative installed wind capacity

Onshore wind farm which is one of the most advanced renewable technologies has proven its commercial viability and made its contributions to reducing climate-change effects and meeting national energy goals. However, compared to the wind farms on land, the offshore environment can offer better locations for wind farms which have better wind profiles for example up to $0.5 \mathrm{~m} / \mathrm{s}$ higher for $10 \mathrm{~km}$ offshore distance [6], less noise and landscape concerns. It's known that the power output from the wind turbine is given by the following expression:

$$
\mathrm{P}=\frac{1}{2} \mathrm{C}_{\mathrm{p}} \rho A U^{3}
$$

Where $C_{p}$ is the power coefficient, $\rho$ is the air density $\left(1.225 \mathrm{~kg} / \mathrm{m}^{3}\right), \mathrm{A}$ is the rotor swept area and $\mathrm{U}$ is the wind speed. From the equation, it can be seen that the change of doubling of wind speed is leading to an eightfold increase in the power output. This cube function between the power output and the wind speed leads to a big attraction of the offshore wind farms. According to the carbon trust study, the UK will need to build 29GW of offshore wind by 2020 to meet the EU'S renewable energy and long term carbon emission targets [7]. Currently the total capacity of offshore with the connection agreements on place with National Grid is $35.2 \mathrm{GW}$ reported by the crown estate [8].

For conventional HVDC interconnector, the ac output from each turbine needs a medium voltage (MV) transformer to boost the low voltage level to a MV collection point and a high voltage (HV) transformer to connect to the HVDC link. 
The use of a DC collection network can improve efficiency and reduce component sizes in the offshore power network. DC-DC converters can eliminate the large line-frequency transformer and increase the efficiency. The proposed offshore wind farm configuration is presented in section 2 which offers a review of key elements in the proposed offshore wind farm configuration. And section 3 presents series-parallel connection of the modular DC-DC converter. In section 4 , the performance of the proposed circuit is investigated through the proposed simulation model. Section 5 considers avenues for future work and summarises the findings of the paper.

\section{Offshore wind farm configuration}

To maximise the utilization of the abundant offshore wind resources, the size of the wind turbine grows rapidly with turbine ratings approaching $10 \mathrm{MW}$ or more. With the increasing size and mass of the wind turbine, reliability is becoming a problem. In this study, the proposed offshore wind farm configuration is based on the permanent magnet synchronous generation and the MV DC collection network.

\subsection{Permanent magnet synchronous generation}

Considering the types of generator, for offshore generation, the new significant commercial interest is based on more reliable and powerful permanent magnet synchronous generator (PMSG) [9]. For example Vestas, GE Wind, Goldwind, Siemens and Gamesa have all introduced the generators with the feature of PMSGs intended for offshore [3]. Permanent magnet synchronous generators are chosen because of the higher reliability, better efficiency, larger range of working conditions and their better power density and energy yield than other types of generators [10]. This configuration can be a wind turbine with a direct drive generator or geared drive type. For direct drive generator, the increased efficiency and reliability by omitting the gearbox give better performances with respect to the energy yield per cost [11]. Moreover, wind turbine with permanent magnet synchronous generator and full size back-to-back converter can provide better performances when grid faults occur [12].

\subsection{DC collection grid}

With the increases of the offshore projects, the problems of offshore wind farm connection to the onshore grid are receiving much attention. In addition, the decreasing cost of power electronics, roughly a factor of 10 over past 10 years [11], nowadays has increased the interest in using HVDC technology and more DC transmission and distribution systems over AC system [13].

The local wind farm grid connects the individual wind turbine unit to the common collecting point, and the collection grid can be realized either through an $\mathrm{AC}$ or a $\mathrm{DC}$ solution as shown in figure 2. The DC grid is the preferred choice when connecting the offshore wind farm for several subsections: Firstly, DC cable is not affected by cable charging current, so that VAR compensators are not required, thus, longer distances can be covered by DC cables. Additionally, compared to the conventional AC based collection network, the heavy line frequency transformer is replaced with high frequency DC-DC converters which lead to significant reduction in the footprint size and weight of the wind turbine and the platform. The DC grid decouples the wind turbine from the AC public grid which enhances the fault withstand capability [14]. Also two-stage DC-DC power convertor covers a very wide range of the DC link voltage on the turbine side to HVDC side etc. For offshore girds with large machines will cover an area leading to longer interconnecting cables were AC charging and losses may be significant.

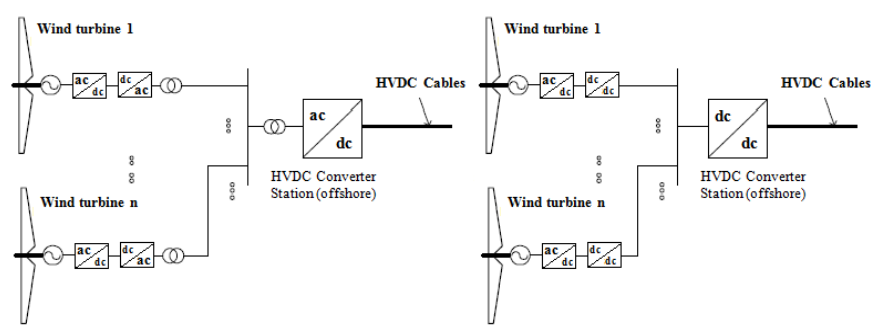

Figure 2: Offshore wind farm AC and DC collection grid

\section{Series-parallel DC-DC converter connection}

For a megawatt-level system, the DC-DC converter is better to be achieved by using several DC-DC elementary modular converters associated with series-parallel connection due to the power capability limitations of the semiconductor devices. The series-parallel converter connection means that the multiple standardized modules are connected in series or parallel at the output and input sides. Normally it is classified into four common architectures based on the connection forms, input-parallel output-parallel (IPOP), input-parallel output-series (IPOS), input-series output-parallel (ISOP), and input-series output-series (ISOS). Series connection is required to match device rating to the required connection voltage whilst parallel connection achieves current sharing and increases capacity. Here, the IPOS connection is chosen to step up the voltage from a low input to a high output application. Full bridge DC-DC converter, which is the association of a full-wave inverter based on IGBT technology, a medium or high switching frequency (several $\mathrm{kHz}$ ) transformer and rectifiers, is chosen here to boost to a desired voltage output because of low component stresses and high power density features. The high switching frequency dramatically reduces the size of the passive components, e.g. filters and transformers. Consequently, the smaller size of the transformer can be applied. In addition, its high frequency transformer prevents fault propagation by providing a galvanic isolation between the source and grid and enables a high output/input voltage ratio. Compared to the conventional DC-DC converter unit, each module here integrates the full bridge DC-DC converter by means of IPOS connection to give a higher step up voltage by output-series configuration [15]. The configuration of IPOS is shown as figure 3. 


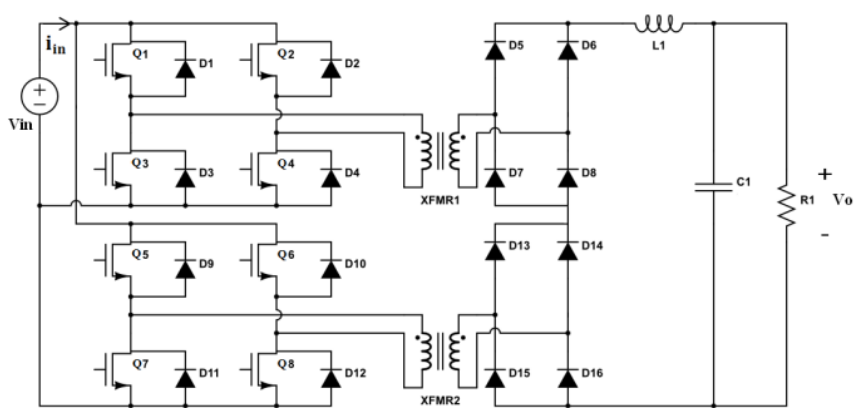

Figure 3: IPOS connection of full bridge modules with a common filter in the output side

\section{Circuit performance analysis}

The benefits of the DC collection gird essentially depend on the performances of the power converters. The feasibility of the converter may be questioned by its losses, saturation and the transformer's harmonics [16]. In this section, the losses of the different DC-DC converters are investigated through the Simulink model.
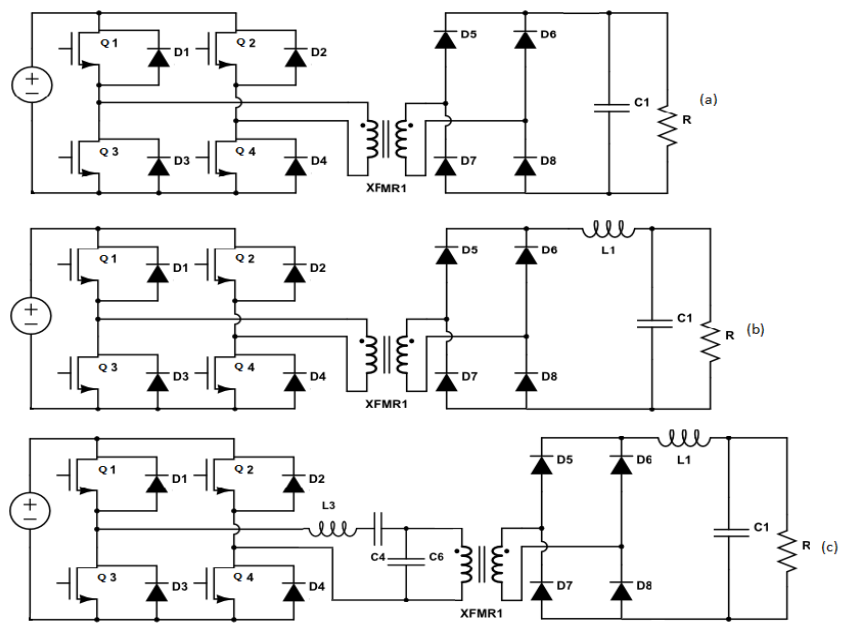

Figure 4: Proposed topologies of DC-DC converter

\subsection{Power loss estimation}

\section{A. Switching losses}

The switching losses in IGBTs are the sum of the turn-on and turn-off losses. While the freewheeling diode losses only count the recovery losses during turn-off and the turn-on energy can be disregarded. From the data sheet, the curve between the switching on and off energy $\left(E_{\text {on }}, E_{\text {off }}\right)$ and the collector current $\left(\mathrm{I}_{\mathrm{c}}\right)$ can be constructed by a fitting polynomial function shown in equation 2 to compute each point value. Therefore, the switching losses can be calculated as a function of the collector current and the switching frequency.

$$
\begin{gathered}
\mathrm{E}_{\mathrm{sw}}=\left(\mathrm{a}+\mathrm{b} \times \mathrm{I}_{\mathrm{c}}+\mathrm{c} \times \mathrm{I}_{\mathrm{c}}^{2}\right) \\
\mathrm{P}_{\mathrm{sw}}=\mathrm{f}_{\mathrm{sw}} \times \mathrm{E}_{\mathrm{sw}}=\mathrm{f}_{\mathrm{sw}}\left(\mathrm{a}+\mathrm{b} \times \mathrm{I}_{\mathrm{c}}+\mathrm{c} \times \mathrm{I}_{\mathrm{c}}^{2}\right)
\end{gathered}
$$

B. Conduction losses
The power dissipation of IGBT and freewheeling diode during the on-state is depending on the on-state voltage drop $\left(\mathrm{V}_{\mathrm{on}}\right)$ and the collector current of IGBT or forward current of diodes, and the voltage drop expresses as:

$$
\mathrm{V}_{\text {on }}=\mathrm{V}_{0}+\mathrm{R}_{\text {on }} \times \mathrm{I}
$$

Where $V_{0}$ is the no load voltage, and $R_{\text {on }}$ is the on-state resistance. Hence, the conduction losses of IGBT can be calculated by the duty cycle (D) and the IGBT on-state characteristics:

$$
\mathrm{P}_{\text {cond }}=\left(\mathrm{V}_{\text {on }} \times \mathrm{I}_{\mathrm{c}}\right) \times \mathrm{D}=\left(\mathrm{V}_{0} \times \mathrm{I}_{\mathrm{c}}+\mathrm{R}_{\text {on }} \times \mathrm{I}_{\mathrm{c}}^{2}\right) \times \mathrm{D}
$$

\subsection{Performance evaluations and simulations}

The case study is using different DC-DC converter topologies with reduced transformer volume due to the high frequency $(10 \mathrm{kHz})$ operation which is achieved by nano-crystalline core material. The table 1 shows the converter parameter values. In this study, ABB IGBT module of "5SNE 0800M170100" is chosen as the example to validate the calculation function. The idea of the loss calculation function is approached by using Simulink. It is noteworthy that, in Simulink all the components are assumed as ideal. So additional function is proposed here to calculate the switching losses and conduction losses. In particular, both continuous and discrete time steps can be applied with thermal consideration. For the conduction loss calculation, a 2-D lookup table which represents the relationship between collector current and temperature is used to get the on-state voltage drop. As shown before, the on-state losses are a function of on-state voltage drop and collector current. Similarly, a 3-D lookup table related to collector current, collector-emitter voltage and switching energy can be applied to get the energy loss. Here, the switching energies of the IGBT are calculated by the given fitting polynomial function, which is shown by equation 2. Figure 5 below presents the layout of the Simulink model, emphasizing the calculation of IGBT losses.

$$
\mathrm{E}_{\mathrm{sw}}=\left(\begin{array}{c}
97.7 \times 10^{-3}+280 \times 10^{-6} \times \mathrm{I}_{\mathrm{c}}+347 \times \\
\left.10^{-9} \times \mathrm{I}_{\mathrm{c}}^{2}\right)
\end{array}\right.
$$

\begin{tabular}{|c|c|c|c|}
\hline Parameter & Value & Parameter & Value \\
\hline $\begin{array}{c}\text { Input supply } \\
\text { voltage }\end{array}$ & $900 \mathrm{~V}$ & $\begin{array}{c}\text { Output load } \\
\text { voltage }\end{array}$ & $1800 \mathrm{~V}$ \\
\hline Full load current & $300 \mathrm{~A}$ & IGBT rating & $1.36 \mathrm{MVA}$ \\
\hline $\begin{array}{c}\text { Transformer } \\
\text { ratio }\end{array}$ & $1: 2$ & $\begin{array}{c}\text { IGBT switching } \\
\text { frequency }\end{array}$ & $10 \mathrm{kHz}$ \\
\hline
\end{tabular}

Table 1: Converter parameter values

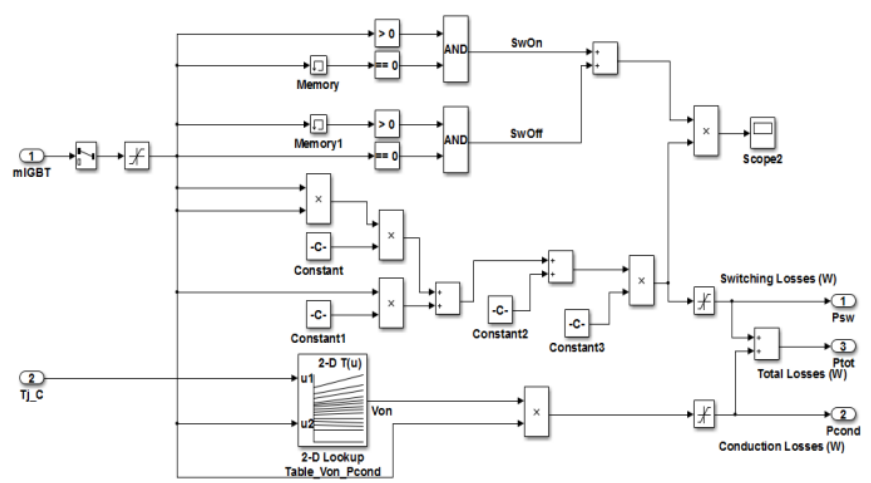

Figure 5: IGBT loss calculation 


\begin{tabular}{|c|c|c|c|}
\hline Loss & Converter a & Converter b & Converter c \\
\hline $\begin{array}{c}\text { Switching } \\
\text { loss(W) }\end{array}$ & 2740 & 2255 & 1880 \\
\hline $\begin{array}{c}\text { Condition } \\
\text { loss(W) }\end{array}$ & 860 & 626 & 565 \\
\hline $\begin{array}{c}\text { Total } \\
\text { converter } \\
\text { loss (\%) }\end{array}$ & 2.67 & 2.13 & 1.72 \\
\hline
\end{tabular}

Table 2: Power loss calculation

It's known that the high switching operation considerably reduces the size and weight, however, the switching losses followed by is a big concern. Converter a is a single active bridge converter which has a voltage stiff output filter. Hence, the current waveform depends on voltage across the transformer leakage inductance. This triangular current waveform results in a high switching loss. Topology $b$ is the full bridge converter. Compared to converter a, it has better loss performances due to the current stiff output filter. For converter $\mathrm{c}$, the switching losses have been minimized by using the soft switching techniques to reduce the loss during the commutation process by the resonant elements. Table 2 reports the losses of different DC-DC converter topologies. As it shown, the resonant DC-DC converter offers slightly better efficiency at the cost of having extra parasitic components. So considering the losses, the complexity of design and control strategy, the full bridge topology is the most suitable choice for offshore collection system.

\section{Conclusions}

This paper has proposed an approach for the loss calculation of the converter under Simulink. Three different topologies of the DC-DC converter are investigated here to check the performance. The results show that the full bridge DC-DC converter is a suitable choice due to the small losses and simple design. The proposed model of single IGBT with loss calculation is found to be successful in dealing with simulating different topologies. The reliability and flexibility have been tested and verified. In future work, the IPOS connection will be applied to the chosen DC-DC converter and the model can be extended to have more control strategies to improve the performances.

\section{Acknowledgements}

The financial support for this project given by engineering and physical sciences research council (EPSRC) is gratefully acknowledged.

\section{References}

1. Renewable_Energy_Technology_Roadmap 20\% by 2020, European Renewable Energy Council (EREC): Brussels.

2. UK Renewable Energy Roadmap Update 2012, 2012, Department of Energy \& Climate Change.
3. Semken, R.S., et al., Direct-drive permanent magnet generators for high-power wind turbines: benefits and limiting factors. Renewable Power Generation, IET, 2012. 6(1): p. 1-8.

4. Robinson, M., Veers, P., Butterfield, S., Greene, J., Thresher, W.M.R., U. DOE, 20\% wind energy by 2030: increasing wind energy's contribution to U.S. electricity supply, 2008, National Renewable Energy Laboratory (NREL), US. DOE.

5. Global Wind Statistics 2012, 2013, Global Wind Energy Council (GWEC): Brussels, Belgium.

6. Bureau, R.E.E., Wind Energy Fact Sheet, 1999, Department for Business Innovation \& Skills: Oxfordshire.

7. Offshore wind power: big challenge, big opportunity, 2008, Carbon Trust.

8. CBE, A.N., UK offshore wind report 2012, 2012, The Crown Estate: London.

9. Polikarpova, M., et al. Thermal design and analysis of a direct-water cooled direct drive permanent magnet synchronous generator for high-power wind turbine application. in Electrical Machines (ICEM), 2012 XXth International Conference on. 2012.

10. Monjean, P., et al. Control strategies of DC-based offshore wind farm. in Power Electronics and Applications (EPE 2011), Proceedings of the 201114th European Conference on. 2011.

11. Li, H. and Z. Chen, Overview of different wind generator systems and their comparisons. Renewable Power Generation, IET, 2008. 2(2): p. 123-138.

12. Bing, L., P. Xiaoran, and T. Undeland. Centralized power control strategy of offshore wind farm with permanent magnetic generators. in Power Electronics and Motion Control Conference, 2009. IPEMC '09. IEEE 6th International. 2009.

13. Carmeli, M.S., et al. MVDC connection of offshore wind farms to the transmission system. in Power Electronics Electrical Drives Automation and Motion (SPEEDAM), 2010 International Symposium on. 2010.

14. Wu, C., et al. A comparison of medium voltage high power DC/DC converters with high step-up conversion ratio for offshore wind energy systems. in Energy Conversion Congress and Exposition (ECCE), 2011 IEEE. 2011.

15. Yi, Z., et al. An active clamp ZVT converter with input-parallel and output-series configuration. in Applied Power Electronics Conference and Exposition (APEC), 2010 Twenty-Fifth Annual IEEE. 2010.

16. Robinson, J., et al., Analysis and Design of an Offshore Wind Farm Using a MV DC Grid. Power Delivery, IEEE Transactions on, 2010. 25(4): p. 2164-2173. 